\title{
Neutrino Mass Models and Leptogenesis
}

\author{
S. F. King ${ }^{1, *}$ \\ ${ }^{1}$ School of Physics and Astronomy, University of Southampton, \\ Southampton, SO17 1BJ, United Kingdom, \\ E-mail: sfk@hep.phys.soton.ac.uk
}

\begin{abstract}
In this talk we show how a natural neutrino mass hierarchy can follow from the type I see-saw mechanism, and a natural neutrino mass degeneracy from the type II see-saw mechanism, where the bi-large mixing angles can arise from either the neutrino or charged lepton sector. We summarize the phenomenological implications of such natural models, and discuss the model building applications of the approach, focussing on the $S U(3) \times S O(10)$ model. We also show that in such type II models the leptogenesis asymmetry parameter becomes proportional to the neutrino mass scale, in sharp contrast to the type I case, which leads to an upper bound on the neutrino mass scale, allowing lighter right-handed neutrinos and hence making leptogenesis more consistent with the gravitino constraints in supersymmetric models.
\end{abstract}

Based on invited talks presented at the 10th International Symposium on Particles, Strings and Cosmology (Pascos04), Northeastern University, Boston, August 16-22, 2004 and Nobel Symposium 129 on Neutrino Physics, Haga Slott, Enkoping, Sweden, August 19-24, 2004.

\footnotetext{
*Email address: sfk@hep.phys.soton.ac.uk
} 


\section{Introduction}

The discovery of neutrino mass and mixing at the end of the last century implies that the Standard Model is incomplete and needs to be extended, but how [1]? In attempting to answer this question, it is useful to being by classifying models in terms of the mechanisms responsible for small neutrino mass, and large lepton mixing, as a first step towards finding the Next Standard Model. Amongst the most elegant mechanisms for small neutrino mass is the see-saw mechanism [2]. However the see-saw mechanism by itself does not provide an explanation for bi-large lepton mixing for either hierarchical or denegerate neutrinos.

In this talk we discuss model independent approaches to accounting for bi-large mixing in a natural way, based on the see-saw mechanism, which are valid for both hierarchical or denegenerate neutrino mass spectra. For the case of hierarchical neutrino masses arising from the type I see-saw mechanism, it is shown how the neutrino mass hierarchy and bi-large mixing angles could originate from the sequential dominance of right-handed neutrinos [3]. It is then shown how to obtain partially degenerate neutrinos in a natural way by including a type II contribution proportional to the unit mass matrix, with the neutrino mass splittings and mixing angles controlled by type I contributions and sequential dominance [4]. The bi-large mixing angles could originate either from the neutrino or the charged lepton sector [5]. For a review see []. We summarize the phenomenological implications of such natural models, and discuss the model building applications of the approach, focussing on the $S U(3) \times S O(10)$ model. We also discuss leptogenesis in such type II models. The leptogenesis asymmetry parameter becomes proportional to the neutrino mass scale, in sharp contrast to the type I case, which leads to an upper bound on the neutrino mass scale, allowing lighter right-handed neutrinos and hence making leptogenesis more consistent with the gravitino constraints in supersymmetric models [7].

\section{See-Saw Mechanism}

The most commonly discussed version of the see-saw mechanism is sometimes called the type I see-saw mechanism [2]. The type I see-saw mechanism is illustrated diagramatically in Fig. 1(a)

In models with a left-right symmetric particle content like minimal left-right symmetric models, PatiSalam models or grand unified theories (GUTs) based on $\mathrm{SO}(10)$, the type I see-saw mechanism is often generalized to a type II see-saw (see e.g. [] $)$, where an additional direct mass term $m_{\mathrm{LL}}^{\mathrm{II}}$ for the light neutrinos is present.

With such an additional direct mass term, the general neutrino mass matrix is given by

$$
\left(\begin{array}{ll}
\overline{\nu_{\mathrm{L}}} & \overline{\nu_{\mathrm{R}}^{\mathrm{C}}}
\end{array}\right)\left(\begin{array}{ll}
m_{\mathrm{LL}}^{\mathrm{II}} & m_{\mathrm{LR}}^{\nu} \\
m_{\mathrm{LR}}^{\nu T} & M_{\mathrm{RR}}
\end{array}\right)\left(\begin{array}{c}
\nu_{\mathrm{L}}^{\mathrm{C}} \\
\nu_{\mathrm{R}}
\end{array}\right) .
$$

Under the assumption that the mass eigenvalues $M_{\mathrm{R} i}$ of $M_{\mathrm{RR}}$ are very large compared to the components of $m_{\mathrm{LL}}^{\mathrm{II}}$ and $m_{\mathrm{LR}}$, the mass matrix can approximately be diagonalized yielding effective Majorana masses

$$
m_{\mathrm{LL}}^{\nu} \approx m_{\mathrm{LL}}^{\mathrm{II}}+m_{\mathrm{LL}}^{\mathrm{I}}
$$




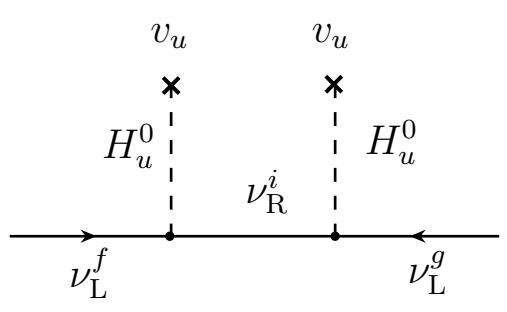

(a)

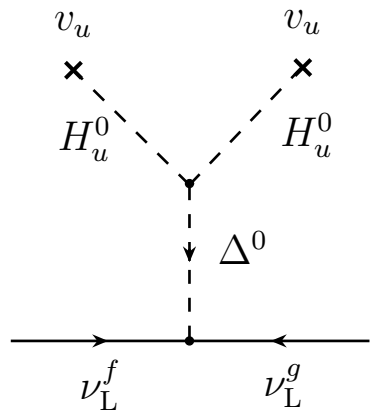

(b)

Figure 1: Diagram (a) shows the contribution from the exchange of a heavy right-handed neutrino as in the type I see-saw mechanism. Diagram (b) illustrates the contribution from an induced vev of the triplet $\Delta$. At low energy, they can be viewed as contributions to the effective neutrino mass operator from integrating out the heavy fields $\nu_{\mathrm{R}}^{i}$ and $\Delta^{0}$, respectively.

with

$$
m_{\mathrm{LL}}^{\mathrm{I}} \approx-m_{\mathrm{LR}}^{\nu} M_{\mathrm{RR}}^{-1} m_{\mathrm{LR}}^{\nu T}
$$

for the light neutrinos. The direct mass term $m_{\mathrm{LL}}^{\mathrm{II}}$ can also provide a naturally small contribution to the light neutrino masses if it stems e.g. from a see-saw suppressed induced vev. The type II contribution may be induced via the exchange of heavy Higgs triplets of $\mathrm{SU}(2)_{\mathrm{L}}$ as illustrated diagramatically in Fig. 1(b)

\section{A Natural Neutrino Mass Hierarchy}

In this section we discuss an elegant and natural way of accounting for a neutrino mass hierarchy and two large mixing angles in the type I see-saw mechanism. The starting point is to assume that one of the right-handed neutrinos contributes dominantly to the see-saw mechanism and determines the atmospheric neutrino mass and mixing. A second right-handed neutrino contributes sub-dominantly and determines the solar neutrino mass and mixing. The third right-handed neutrino is effectively decoupled from the see-saw mechanism.

The above Sequential Dominance mechanism [3] is most simply described assuming three right-handed neutrinos in the basis where the right-handed neutrino mass matrix is diagonal although it can also be developed in other bases. In this basis we write the input see-saw matrices as

$$
M_{\mathrm{RR}}=\left(\begin{array}{ccc}
X & 0 & 0 \\
0 & Y & 0 \\
0 & 0 & Z
\end{array}\right), \quad m_{\mathrm{LR}}^{\nu}=\left(\begin{array}{ccc}
a & d & p \\
b & e & q \\
c & f & r
\end{array}\right)
$$

Each right-handed neutrino in the basis of Eq⿴囗十 couples to a particular column of $m_{\mathrm{LR}}^{\nu}$. There is no mass ordering of $X, Y, Z$ implied in Eq廿 The dominant right-handed neutrino may be taken to be the one with mass $Y$ without loss of generality. Sequential dominance occurs when the right-handed neutrinos dominate sequentially [3]:

$$
\frac{\left|e^{2}\right|,\left|f^{2}\right|,|e f|}{Y} \gg \frac{|x y|}{X} \gg \frac{\left|x^{\prime} y^{\prime}\right|}{Z}
$$


where $x, y \in a, b, c$ and $x^{\prime}, y^{\prime} \in p, q, r$. This leads to a full neutrino mass hierarchy $m_{3}^{2} \gg m_{2}^{2} \gg m_{1}^{2}$. Ignoring phases, in the case that $d=0$, corresponding to a Yukawa 11 texture zero in Eq4 we have:

$$
m_{1} \sim O\left(\frac{x^{\prime} y^{\prime}}{Z}\right), \quad m_{2} \approx \frac{|a|^{2}}{X s_{12}^{2}}, \quad m_{3} \approx \frac{|e|^{2}+|f|^{2}}{Y}
$$

where $s_{12}=\sin \theta_{12}$ is given below. Note that each neutrino mass is generated by a separate right-handed neutrino, and the sequential dominance condition naturally results in a neutrino mass hierarchy $m_{1} \ll m_{2} \ll$ $m_{3}$. The neutrino mixing angles are given to leading order in $m_{2} / m_{3}$ by 3 :

$$
\tan \theta_{23} \approx \frac{e}{f}, \quad \tan \theta_{12} \approx \frac{a}{c_{23} b-s_{23} c}, \quad \theta_{13} \approx s_{12} c_{12} \frac{\left(s_{23} b+c_{23} c\right)}{\left(c_{23} b-s_{23} c\right)} \frac{m_{2}}{m_{3}}
$$

Physically these results show that in sequential dominance the atmospheric neutrino mass $m_{3}$ and mixing $\theta_{23}$ is determined by the couplings of the dominant right-handed neutrino of mass $Y$. The angle $\theta_{13}$ is generically of order $\theta_{13} \sim O\left(m_{2} / m_{3}\right) \sim 0.2$. However the coefficient in Eq[7 can be arbitrarily small, since to leading order as $b \rightarrow-c, \theta_{13} \rightarrow 0$, but $\theta_{12}$ remains large. The solar neutrino mass $m_{2}$ and mixing $\theta_{12}$ is determined by the couplings of the sub-dominant right-handed neutrino of mass $X$. From Eq 7 the solar angle only depends on the sub-dominant couplings and the simple requirement for large solar angle is $a \sim b-c$. The third right-handed neutrino of mass $Z$ is effectively decoupled from the see-saw mechanism and leads to the vanishingly small mass $m_{1} \approx 0$.

\section{A Natural Neutrino Mass Degeneracy}

We now show that it is possible to obtain a (partially) degenerate neutrino mass spectrum by essentially adding a type II direct neutrino mass contribution proportional to the unit matrix: Thus we shall consider a type II extension [4, where the mass matrix of the light neutrinos has the form:

$$
m_{\mathrm{LL}}^{\nu} \approx m^{\mathrm{II}}\left(\begin{array}{lll}
1 & 0 & 0 \\
0 & 1 & 0 \\
0 & 0 & 1
\end{array}\right)+m_{\mathrm{LL}}^{\mathrm{I}}
$$

Assuming here that the type I mass matrix $m_{\mathrm{LL}}^{\mathrm{I}}$ is real, the full neutrino mass matrix is diagonalised by the same matrix that diagonalises the type I matrix:

$$
\left(m_{\mathrm{LL}}^{\nu}\right)_{\operatorname{diag}}=m^{\mathrm{II}} V V^{T}+V m_{\mathrm{LL}}^{\mathrm{I}} V^{T}=m^{\mathrm{II}} \mathbb{1}+\operatorname{diag}\left(m_{1}^{\mathrm{I}}, m_{2}^{\mathrm{I}}, m_{3}^{\mathrm{I}}\right) .
$$

In this case the neutrino mass scale is controlled by the type II mass scale $m^{\mathrm{II}}$, while the neutrino mass splittings are determined by the type I mass eigenvalues:

$$
m_{1} \approx\left|m^{\mathrm{II}}-m_{2}^{\mathrm{I}}\right|, \quad m_{2} \approx\left|m^{\mathrm{II}}-m_{2}^{\mathrm{I}}\right|, \quad m_{3} \approx\left|m^{\mathrm{II}}-m_{3}^{\mathrm{I}}\right| .
$$

Sequential dominance in the type I sector naturally predicts $m_{1}^{\mathrm{I}} \ll m_{2}^{\mathrm{I}} \ll m_{3}^{\mathrm{I}}$. Hence the very small mass splittings required for degenerate neutrinos can be achieved naturally by sequential dominance. The predictions for the mixings are determined from the type I mass matrix (the type II unit matrix is irrelevant). In particular the atmospheric and solar angles are given by the sequential dominance estimates in Eq[7 and are independent of the type II neutrino mass scale $m^{\mathrm{II}}$. However the angle $\theta_{13}$ in Eq[7 is now of order $\theta_{13} \sim O\left(m_{2}^{\mathrm{I}} / m_{3}^{\mathrm{I}}\right)$. This is now much smaller than the type I result $\theta_{13} \sim O\left(m_{2} / m_{3}\right)$ since the neutrino mass splittings, controlled by the type I masses, are very small for partially degenerate neutrinos. 


\section{$5 \quad$ Mixing Angles From the Charged Leptons?}

In this section we show how bi-large mixing could originate from the charged lepton sector using a generalization of sequential right-handed neutrino dominance to all right-handed leptons [5]. We write the mass matrices for the charged leptons $m_{\mathrm{E}}$ as

$$
m_{\mathrm{E}}=\left(\begin{array}{ccc}
p^{\prime} & d^{\prime} & a^{\prime} \\
q^{\prime} & e^{\prime} & b^{\prime} \\
r^{\prime} & f^{\prime} & c^{\prime}
\end{array}\right) .
$$

In our notation, each right-handed charged lepton couples to a column in $m_{\mathrm{E}}$. For the charged leptons, the sequential dominance conditions are [5]:

$$
\left|a^{\prime}\right|,\left|b^{\prime}\right|,\left|c^{\prime}\right| \gg\left|d^{\prime}\right|,\left|e^{\prime}\right|,\left|f^{\prime}\right| \gg\left|p^{\prime}\right|,\left|q^{\prime}\right|,\left|r^{\prime}\right| .
$$

They imply the desired hierarchy for the charged lepton masses $m_{\tau} \gg m_{\mu} \gg m_{e}$ and small right-handed mixing of $U_{e_{\mathrm{R}}}$. We assume zero mixing from the neutrino sector. A natural possibility for obtaining a small $\theta_{13}$ is [5]

$$
\left|d^{\prime}\right|,\left|e^{\prime}\right| \ll\left|f^{\prime}\right| .
$$

In leading order in $\left|d^{\prime}\right| /\left|f^{\prime}\right|$ and $\left|e^{\prime}\right| /\left|f^{\prime}\right|$, for the mixing angles $\theta_{12}, \theta_{23}$ and $\theta_{13}$, we obtain (again ignoring phases here)

$$
\tan \theta_{12} \approx \frac{a^{\prime}}{b^{\prime}}, \quad \tan \theta_{23} \approx \frac{s_{12} a^{\prime}+c_{12} b^{\prime}}{c^{\prime}}, \quad \tan \theta_{13} \approx \frac{s_{12} e^{\prime}-c_{12} d^{\prime}}{f^{\prime}}
$$

$\theta_{13}$ only depends on $d^{\prime} / f^{\prime}$ and $e^{\prime} / f^{\prime}$ from the Yukawa couplings to the sub-dominant right-handed muon and on $\theta_{12}$. We find that in the limit $\left|d^{\prime}\right|,\left|e^{\prime}\right| \ll\left|f^{\prime}\right|$, the two large mixing angles $\theta_{12}$ and $\theta_{23}$ approximately depend only on $a^{\prime} / c^{\prime}$ and $b^{\prime} / c^{\prime}$ from the right-handed tau Yukawa couplings. Both mixing angles are large if $a^{\prime}, b^{\prime}$ and $c^{\prime}$ are of the same order.

\section{Phenomenological Implications}

We now summarize the phenomenological consequences of type I see-saw models with a natural mass hierarchy and their type II extensions with a natural mass degeneracy, for the low energy neutrino parameters and high-energy mechanisms as leptogenesis and minimal lepton flavour violation (LFV). In order to compare the predictions of such natural see-saw models (based on sequential dominance) with the experimental data obtained at low energy, the renormalization group (RG) running of the effective neutrino mass matrix has to be taken into account.

\subsection{Renormalization Group Corrections}

For type I models with sequential dominance, the running of the mixing angles is generically small [14] since the mass scheme is strongly hierarchical. When the neutrino mass scale is lifted, e.g. via a type II upgrade, a careful treatment of the RG running of the neutrino parameters, including the energy ranges between and above the see-saw scale [14], is required. Dependent on $\tan \beta$ in the MSSM, on the size of the neutrino Yukawa couplings and on the neutrino mass scale, the RG effects can be sizable or cause only small corrections. 


\subsection{Dirac and Majorana CP Phases and Neutrinoless Double Beta Decay}

At present, the $\mathrm{CP}$ phases in the lepton sector are unconstrained by experiment. In type I see-saw models based on sequential dominance, there is no restriction on them from a theoretical point of view. The typeII-extension however predicts that all observable CP phases, i.e. the Dirac CP phase $\delta$ relevant for neutrino oscillations and the Majorana CP phases $\beta_{2}$ and $\beta_{3}$, become small as the neutrino mass scale increases.

The key process for measuring the neutrino mass scale could be neutrinoless double beta decay. The decay rates depend on an effective Majorana mass defined by $\left\langle m_{\nu}\right\rangle=\left|\sum_{i}\left(U_{\mathrm{MNS}}\right)_{1 i}^{2} m_{i}\right|$. Future experiments which are under consideration at present might increase the sensitivity to $\left\langle m_{\nu}\right\rangle$ by more than an order of magnitude. For type I models with sequential dominance, which have a hierarchical mass scheme, $\left\langle m_{\nu}\right\rangle$ can be very small, below the accessible sensitivity.

For models where the neutrino mass scale is lifted via a type II extension 4], there is a close relation between the neutrino mass scale, i.e. the mass of the lightest neutrino and $\left\langle m_{\nu}\right\rangle$. Since the CP phases are small, there can be no significant cancellations in $\left\langle m_{\nu}\right\rangle$. This implies that the effective mass for neutrinoless double beta decay is approximately equal to the neutrino mass scale $\left\langle m_{\nu}\right\rangle \approx m^{\mathrm{II}}$ and therefore neutrinoless double beta decay will be observable in the next round of experiments if the neutrino mass spectrum is partially degenerate.

\subsection{Theoretical expectations for the Mixing Angles}

In order to discriminate between models, precision measurements of the neutrino mixing angles have the potential to play an important role.

One important parameter is the value of the mixing angle $\theta_{13}$, which is at present only bounded from above to be smaller than approximately $13^{\circ}$. In the type I sequential dominance case, the mixing angle $\theta_{13}$ is typically of the order $\mathcal{O}\left(m_{2}^{\mathrm{I}} / m_{3}^{\mathrm{I}}\right)$. In the type-II-upgrade scenario this ratio decreases with increasing neutrino mass scale and is smaller than $\approx 5^{\circ}$ for partially degenerate neutrinos even if it was quite large in the type I limit. Sizable RG corrections, which are usually expected for partially degenerate neutrinos, are suppressed in the type-II-upgrade scenario due to small CP phases $\beta_{2}, \beta_{3}$ and $\delta$.

Another important parameter is $\theta_{23}$. Its present best-fit value is close to $45^{\circ}$, however comparably large deviations are experimentally allowed as well. With sequential dominance, we expect minimal deviations of $\theta_{23}$ from $45^{\circ}$ of the order $\mathcal{O}\left(m_{2}^{\mathrm{I}} / m_{3}^{\mathrm{I}}\right)$, which could be observed by future long-baseline experiments in the type I see-saw case. In the type II upgraded version, the corrections can be significantly smaller since the ratio $m_{2}^{\mathrm{I}} / m_{3}^{\mathrm{I}}$ decreases with increasing neutrino mass scale [4]. For large $\tan \beta$ in the MSSM, the major source for the corrections can be RG effects, which are un-suppressed for small CP phases.

\subsection{Minimal Lepton Flavour Violation}

At leading order in a mass insertion approximation the branching fractions of LFV processes are given by ${ }^{\dagger}$

$$
\operatorname{BR}\left(l_{i} \rightarrow l_{j} \gamma\right) \approx \frac{\alpha^{3}}{G_{F}^{2}} f\left(M_{2}, \mu, m_{\tilde{\nu}}\right)\left|m_{\tilde{L}_{i j}}^{2}\right|^{2} \tan ^{2} \beta,
$$

\footnotetext{
${ }^{\dagger}$ The mass insertion approximation given in Eq15 is for illustrative purposes only. The conclusions quoted below from 9 do not rely on this approximation.
} 
where $l_{1}=e, l_{2}=\mu, l_{3}=\tau$, and where the off-diagonal slepton doublet mass squared is given in the leading log approximation (LLA) by

$$
m_{\tilde{L}_{i j}}^{2(L L A)} \approx-\frac{\left(3 m_{0}^{2}+A_{0}^{2}\right)}{8 \pi^{2}} C_{i j}
$$

With sequential dominance, using the notation of Eqs 4 the leading log coefficients relevant for $\mu \rightarrow e \gamma$ and $\tau \rightarrow \mu \gamma$ are given approximately as

$$
\begin{aligned}
& C_{21}=a b \ln \frac{M_{U}}{X}+d e \ln \frac{M_{U}}{Y} \\
& C_{32}=b c \ln \frac{M_{U}}{X}+e f \ln \frac{M_{U}}{Y} .
\end{aligned}
$$

Large rates of $\tau \rightarrow \mu \gamma$ which is the characteristic expectation of lop-sided models in general 9]. Such models occur if the dominant right-handed neutrino is the heaviest one. A global analysis of LFV has been performed in the constrained minimal supersymmetric standard model (CMSSM) for the case of sequential dominance, focussing on the two cases where the dominant right-handed neutrino is either the heaviest one or the lightest one 9. If the dominant right-handed neutrino is the lightest one then the rate for $\tau \rightarrow \mu \gamma$ is well below observable values. Therefore $\tau \rightarrow \mu \gamma$ provides a good discriminator between the two cases of dominance. In [9] it is shown that the rate for $\mu \rightarrow e \gamma$ may determine the order of the sub-dominant neutrino Yukawa couplings in the flavour basis.

\section{Model Building Applications}

\subsection{Effective Two Right-Handed Neutrino Models}

In sequential dominance we have seen that one of the right-handed neutrinos effectively decouples from the see-saw mechanism. If the decoupled right-handed neutrino is also the heaviest then it would be expected to play no part in phenomenology. In this case sequential dominance reduces to effectively two right-handed neutrino models [3]. Recently there have been several studies based on the "minimal see-saw" involving two right-handed neutrinos [10], and it is worth bearing in mind that such models could naturally arise as the limiting case of sequential dominance.

\subsection{Sneutrino Inflation Models}

Sequential dominance has recently also been applied to sneutrino inflation [15], [16. Requiring a low reheat temperature after inflation, in order to solve the gravitino problem, forces the sneutrino inflaton to couple very weakly to ordinary matter and its superpartner almost to decouple from the see-saw mechanism. This decoupling of a right-handed neutrino from the see-saw mechanism is a characteristic of sequential dominance.

\subsection{GUT and Family Symmetry Models}

There are many models in the literature based on sequential dominance. A Pati-Salam model with $U(1)$ family symmetry was considered in [11]. Single right-handed neutrino dominance has also been applied to $\mathrm{SO}(10)$ GUT models involving a $U(2)$ family symmetry [12. Sequential dominance with $S U(3)$ family symmetry and $S O(10)$ GUTs has been considered in [13. Type II up-gradable models based on sequential 
dominance of the ISD type with $S O(3)$ family symmetry have been considered in [5] 4 . For GUT models the renormalisation group corrections need to be taken into account, although for a natural hierarchy such corrections are only a few per cent [14].

As an example of a model based on a non-Abelian family symmetry, we briefly review the model proposed in [17. The model uses the largest family symmetry $S U(3)$ consistent with $S O(10)$ GUTs. An important further motivation for $S U(3)$ family symmetry is, in the framework of sequential dominance, to relate the second and third entries of the Yukawa matrix, as required to obtain an almost maximal 23 mixing in the atmospheric neutrino sector [13. The theoretical requirements that the neutrino Yukawa matrix resembles the quark Yukawa matrices, and therefore has a large 33 element with no large off-diagonal elements and a texture zero in the 11 position leads uniquely to the dominant right-handed neutrino being the first (lightest) one. Assuming this then the atmospheric neutrino mixing angle is given by $\tan \theta_{23}^{\nu} \approx Y_{21}^{\nu} / Y_{31}^{\nu} \approx 1$. The sequential dominance conditions which were assumed earlier will here be derived from the symmetries of the model. Thus this model provides an example of the application of sequential dominance to realistic models of flavour, and shows how the conditions of sequential dominance which were simply assumed earlier can motivate models based on GUTs and family symmetry which are capable of explaining these conditions. In other words, the conditions for sequential dominance can provide clues to the nature of the underlying flavour theory.

The starting point of the model is the observation that an excellent fit to all quark data is given by the approximately symmetric form of quark Yukawa matrices [18]

$$
Y^{u} \propto\left(\begin{array}{ccc}
0 & \epsilon^{3} & O\left(\epsilon^{3}\right) \\
\cdot & \epsilon^{2} & O\left(\epsilon^{2}\right) \\
\cdot & . & 1
\end{array}\right), \quad Y^{d} \propto\left(\begin{array}{ccc}
0 & 1.5 \bar{\epsilon}^{3} & 0.4 \bar{\epsilon}^{3} \\
. & \bar{\epsilon}^{2} & 1.3 \bar{\epsilon}^{2} \\
. & . & 1
\end{array}\right)
$$

where the expansion parameters $\epsilon$ and $\bar{\epsilon}$ are given by

$$
\epsilon \approx 0.05, \quad \bar{\epsilon} \approx 0.15
$$

This motivates a particular model in which the three families are unified as triplets under an $S U(3)$ family symmetry, and $16^{\prime} s$ under an $S O(10)$ GUT [13, 17,

$$
\psi_{i}=(3,16),
$$

where the $S O(10)$ is broken via the Pati-Salam group resulting in:

$$
\psi_{i}=(3,4,2,1), \quad \bar{\psi}_{i}=(3, \overline{4}, 1, \overline{2}) .
$$

Further symmetries $R \times Z_{2} \times U(1)$ are assumed to ensure that the vacuum alignment leads to a universal form of Dirac mass matrices for the neutrinos, charged leptons and quarks [17. To build a viable model we also need spontaneous breaking of the family symmetry

$$
S U(3) \longrightarrow S U(2) \longrightarrow \text { Nothing }
$$

To achieve this symmetry breaking additional Higgs fields $\phi_{3}, \bar{\phi}_{3}, \phi_{23}$ and $\bar{\phi}_{23}$ are required. The largeness of the third family fermion masses implies that $S U(3)$ must be strongly broken by new Higgs antitriplet 
fields $\phi_{3}$ which develop a vev in the third $S U(3)$ component $<\phi_{3}>^{T}=\left(0,0, a_{3}\right)$ as in [13]. $\phi_{3}^{i}$ transforms under $S U(2)_{R}$ as $\mathbf{3} \oplus \mathbf{1}$ rather than being $S U(2)_{R}$ singlets as assumed in [13, and develops vevs in the $S U(3) \times S U(2)_{R}$ directions

$$
<\phi_{3}>=<\overline{\phi_{3}}>=\left(\begin{array}{l}
0 \\
0 \\
1
\end{array}\right) \otimes\left(\begin{array}{cc}
a_{3}^{u} & 0 \\
0 & a_{3}^{d}
\end{array}\right) .
$$

The symmetry breaking also involves the $S U(3)$ antitriplets $\phi_{23}$ which develop vevs [13]

$$
<\phi_{23}>=\left(\begin{array}{l}
0 \\
1 \\
1
\end{array}\right) b,
$$

where, as in [13, vacuum alignment ensures that the vevs are aligned in the 23 direction. Due to D-flatness there must also be accompanying Higgs triplets such as $\overline{\phi_{23}}$ which develop vevs 13

$$
<\overline{\phi_{23}}>=\left(\begin{array}{l}
0 \\
1 \\
1
\end{array}\right) b .
$$

We also introduce an adjoint $\Sigma$ field which develops vevs in the $S U(4)_{P S} \times S U(2)_{R}$ direction which preserves the hypercharge generator $Y=T_{3 R}+(B-L) / 2$, and implies that any coupling of the $\Sigma$ to a fermion and a messenger such as $\Sigma_{b \beta}^{a \alpha} \psi_{a \alpha}^{c} \chi^{b \beta}$, where the $S U(2)_{R}$ and $S U(4)_{P S}$ indices have been displayed explicitly, is proportional to the hypercharge $Y$ of the particular fermion component of $\psi^{c}$ times the vev $\sigma$. In addition a $\theta$ field is required for the construction of Majorana neutrino masses.

The leading operators allowed by the symmetries are

$$
\begin{aligned}
P_{\text {Yuk }} & \sim \frac{1}{M^{2}} \psi_{i} \phi_{3}^{i} \bar{\psi}_{j} \phi_{3}^{j} h \\
& +\frac{\Sigma}{M^{3}} \psi_{i} \phi_{23}^{i} \bar{\psi}_{j} \phi_{23}^{j} h \\
P_{\text {Maj }} & \sim \frac{1}{M} \bar{\psi}_{i} \theta^{i} \theta^{j} \bar{\psi}_{j}
\end{aligned}
$$

where the operator mass scales, generically denoted by $M$ may differ and we have suppressed couplings of $O(1)$.

The final form of the Yukawa matrices and heavy Majorana matrix after inserting a particular choice of order unity coefficients is 17

$$
\begin{aligned}
Y^{u} & \approx\left(\begin{array}{lrr}
0 & 1.2 \epsilon^{3} & 0.9 \epsilon^{3} \\
-1.2 \epsilon^{3} & -\frac{2}{3} \epsilon^{2} & -\frac{2}{3} \epsilon^{2} \\
-0.9 \epsilon^{3} & -\frac{2}{3} \epsilon^{2} & 1
\end{array}\right) \bar{\epsilon} \\
Y^{d} & \approx\left(\begin{array}{llr}
0 & 1.6 \bar{\epsilon}^{3} & 0.7 \bar{\epsilon}^{3} \\
-1.6 \bar{\epsilon}^{3} & \bar{\epsilon}^{2} & \bar{\epsilon}^{2}+\bar{\epsilon}^{\frac{5}{2}} \\
-0.7 \bar{\epsilon}^{3} & \bar{\epsilon}^{2} & 1
\end{array}\right) \bar{\epsilon} \\
Y^{e} \approx & \left(\begin{array}{llr}
0 & 1.6 \bar{\epsilon}^{3} & 0.7 \bar{\epsilon}^{3} \\
-1.6 \bar{\epsilon}^{3} & 3 \bar{\epsilon}^{2} & 3 \bar{\epsilon}^{2} \\
-0.7 \bar{\epsilon}^{3} & 3 \bar{\epsilon}^{2} & 1
\end{array}\right) \bar{\epsilon} \\
Y^{\nu} & \approx\left(\begin{array}{llr}
0 & 1.2 \epsilon^{3} & 0.9 \epsilon^{3} \\
-1.2 \epsilon^{3} & -\alpha \epsilon^{2} & -\alpha \epsilon^{2} \\
-0.9 \epsilon^{3} & -\alpha \epsilon^{2}-\epsilon^{3} & 1
\end{array}\right) \bar{\epsilon} .
\end{aligned}
$$




$$
M_{R R} \approx\left(\begin{array}{ccc}
\epsilon^{6} \bar{\epsilon}^{3} & 0 & 0 \\
0 & \epsilon^{6} \bar{\epsilon}^{2} & 0 \\
0 & 0 & 1
\end{array}\right) M_{3}
$$

The model gives excellent agreement with the quark and lepton masses and mixing angles. For the up and down quarks the form of $Y^{u}$ and $Y^{d}$ given in Eq 29$]$ is consistent with the phenomenological fit in Eq18 The charged lepton mass matrix is of the Georgi-Jarslkog [19] form which, after including radiative corrections, gives an excellent description of the charged lepton masses. In the neutrino sector the parameters satisfy the conditions of sequential dominance in Eq[5] with the lightest right-handed neutrino giving the dominant contribution to the heaviest physical neutrino mass, and the second right-handed neutrino giving the leading subdominant contribution, providing that $\alpha \sim \epsilon$.

Analytic estimates of neutrino masses and mixing angles for sequential dominance were derived in Section 3 [3]. With the dominant right-handed neutrino of mass $Y$ being the lightest one, the matrices in Eq⿴囗4 should be re-ordered as follows before comparing to the neutrino matrices in Eqs 32 and 33

$$
M_{\mathrm{RR}}=\left(\begin{array}{ccc}
Y & 0 & 0 \\
0 & X & 0 \\
0 & 0 & Z
\end{array}\right), \quad m_{\mathrm{LR}}^{\nu}=Y^{\nu} v_{2}=\left(\begin{array}{ccc}
0 & a & p \\
e & b & q \\
f & c & r
\end{array}\right) .
$$

The above re-ordering of course leaves the results in Eqs [ and [ 7 unchanged. The analytic estimates for the neutrino masses are obtained by comparing the matrices in Eqs 34 to those in Eqs 32 and 33 then using the results in Eqs 6 and 7

$$
\begin{aligned}
& m_{1} \sim \bar{\epsilon}^{2} \frac{v_{2}^{2}}{M_{3}} \\
& m_{2} \approx 5.8 \frac{v_{2}^{2}}{M_{3}} \\
& m_{3} \approx 15 \frac{v_{2}^{2}}{M_{3}}
\end{aligned}
$$

and neutrino mixing angles:

$$
\begin{aligned}
\tan \theta_{23}^{\nu} & \approx 1.3 \\
\tan \theta_{12}^{\nu} & \approx 0.66 \\
\theta_{13}^{\nu} & \approx 1.6 \bar{\epsilon}
\end{aligned}
$$

The physical lepton mixing angle $\theta_{13}$ receives a large contribution from the neutrino sector $\theta_{13}^{\nu} \sim 0.3$ at the high energy scale, for this choice of parameters, compared to the current CHOOZ limit $\theta_{13} \leq 0.2$. However the physical mixing angles will receive charged lepton contributions [3] and all the parameters are subject to radiative corrections in running from the high energy scale to low energies, although in sequential dominance models these corrections are only a few per cent [14]. Thus the model predicts that $\theta_{13}$ is close to the current CHOOZ limit, and could be observed by the next generation of long baseline experiments such as MINOS or OPERA. 


\section{Leptogenesis}

Neutrino mass allows the possibility that the baryon asymmetry of the universe is generated by out-ofequilibrium decay of lepton-number violating Majorana right-handed (s)neutrinos, whose decays result in a net lepton number which is subsequently converted to a net baryon number by sphaleron transitions. This mechanism is known as leptogenesis [20]. In models which give a natural neutrino mass hierarchy, if the dominant right-handed neutrino is the lightest one then the washout parameter $\tilde{m}_{1} \sim O\left(m_{3}\right)$, which is rather too large compared to the optimal value of around $10^{-3} \mathrm{eV}$, while if the dominant right-handed neutrino is either the intermediate one or the heaviest one then one finds $\tilde{m}_{1} \sim O\left(m_{2}\right)$ or arbitrary $\tilde{m}_{1}$, which can be closer to the desired value [21].

It is interesting to note that if the dominant right-handed neutrino is the lightest one, and there is a 11 texture zero, as is the case in the $S U(3) \times S O(10)$ model discussed in the last section, then there is a link between the $\mathrm{CP}$ violation required for leptogenesis, $\phi_{\mathrm{COSMO}}$, and the phase $\delta$ measurable in accurate neutrino oscillation experiments [22]. $\delta$ turns out to be a function of the same two see-saw phases that also determine $\phi_{\text {COSMO }}$. If both the see-saw phases are zero, then both $\phi_{\text {COSMO }}$ and $\delta$ are necessarily zero. This feature is absolutely crucial. It means that, barring cancellations, measurement of a non-zero value for the phase $\delta$ at a neutrino factory will be a signal of a non-zero value of the leptogenesis phase $\phi_{\text {Cosmo }}$. We also find the remarkable result

$$
\left|\phi_{\mathrm{COSMO}}\right|=\left|\phi_{\beta \beta 0 \nu}\right|
$$

where $\phi_{\beta \beta 0 \nu}$ is the phase which enters the rate for neutrinoless double beta decay [22].

We now discuss the consequences of the neutrino mass scale for leptogenesis via the out-of-equilibrium decay of the lightest right-handed (s)neutrinos in type II see-saw models [7. In [7] we calculated the type II contributions to the decay asymmetries for minimal scenarios based on the Standard Model (SM) and on the Minimal Supersymmetric Standard Model (MSSM), where the additional direct mass term for the neutrinos stems from the induced vev of a triplet Higgs. The diagrams are shown in Figure 2, The result we obtained for the supersymmetric case is new and we corrected the previous result in the scenario based on the Standard Model. 23].

We subsequently derived a general upper bound on the decay asymmetry and found that it increases with the neutrino mass scale:

$$
\begin{aligned}
\left|\varepsilon_{1}^{\mathrm{SM}}\right| & \leq \frac{3}{16 \pi} \frac{M_{\mathrm{R} 1}}{v_{\mathrm{u}}^{2}} m_{\max }^{\nu}, \\
\left|\varepsilon_{1}^{\mathrm{MSSM}}\right| & \leq \frac{3}{8 \pi} \frac{M_{\mathrm{R} 1}}{v_{\mathrm{u}}^{2}} m_{\max }^{\nu}
\end{aligned}
$$

It leads to a lower bound on the mass of the lightest right-handed neutrino, which is significantly below the type I bound for partially degenerate neutrinos. It is worth emphasizing that these results are in sharp contrast to the type I see-saw mechanism where an upper bound on the neutrino mass scale is predicted. Here we find no upper limit on the neutrino mass scale which may be increased arbitrarily. Indeed we find that the lower bound on the mass of the lightest right-handed neutrino decreases as the physical neutrino 


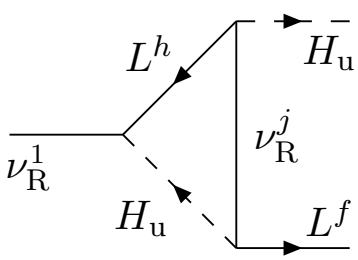

(a)

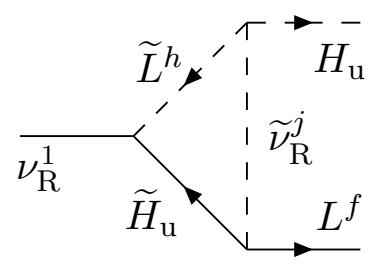

(d)

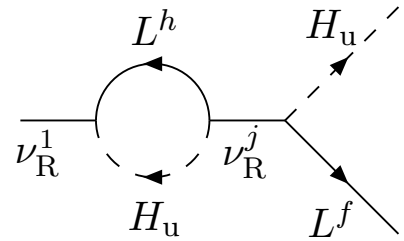

(b)

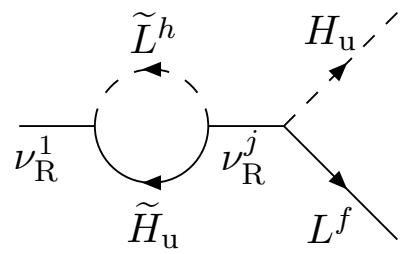

(e)

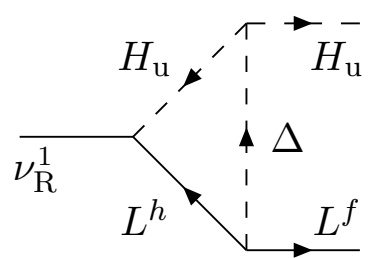

(c)

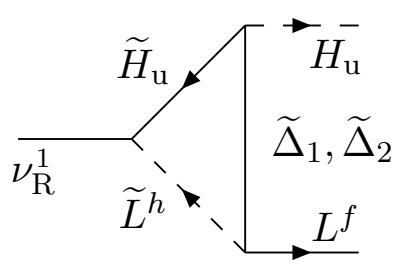

(f)

Figure 2: Loop diagrams in the MSSM which contribute to the decay $\nu_{\mathrm{R}}^{1} \rightarrow L_{a}^{f} H_{\mathrm{u} b}$ for the case of a type II see-saw mechanism where the direct mass term for the neutrinos stems from the induced vev of a Higgs triplet. In diagram (f), $\widetilde{\Delta}_{1}$ and $\widetilde{\Delta}_{2}$ are the mass eigenstates corresponding to the superpartners of the $\mathrm{SU}(2)_{\mathrm{L}}$-triplet scalar fields $\Delta$ and $\bar{\Delta}$. The SM diagrams are the ones where no superpartners (marked by a tilde) are involved and where $H_{\mathrm{u}}$ is renamed to the SM Higgs.

mass scale increases. This allows a lower reheat temperature, making thermal leptogenesis more consistent with the gravitino constraints in supersymmetric models.

\section{Conclusion}

In this talk we have discussed how a natural neutrino mass hierarchy can follow from the type I see-saw mechanism, and a natural neutrino mass degeneracy from the type II see-saw mechanism, where the bi-large mixing angles can arise from either the neutrino or charged lepton sector. The key to achieving naturalness is the idea of sequential dominance of right-handed neutrinos, namely that in the see-saw mechanism one of the right-handed neutrinos dominates and couples with approximately equal strength to the $\tau$ and $\mu$ families, leading to an approximately maximal atmospheric mixing angle. A second right-handed neutrino then plays the leading sub-dominant role, and couples with approximately equal strength to all three families, leading to a large solar mixing angle. We have shown that this can lead either to a hierchical neutrino mass spectrum or, if a type II contribution proportional to the unit matrix is considered, to approximately degenerate neutrino masses. We have summarised the phenomenological implications of such a natural approach to neutrino model building, and have discussed some of the model building applications, focussing on the $S U(3) \times S O(10)$ model. We then turned to leptogenesis and mentioned the possible link between the leptogenesis phase and the phase measurable in neutrino oscillation experiments. We then pointed out that in natural type II models the leptogenesis asymmetry parameter becomes proportional to the neutrino mass scale, in sharp contrast to the type I case, which leads to an upper bound on the neutrino mass scale, allowing lighter right-handed neutrinos and hence making leptogenesis more consistent with the gravitino constraints in supersymmetric models. 
Acknowledgements I would like to thank Stefan Antusch for his collaboration in some of the work presented here. I would also like to thank the organisers of Pascos '04 and the Nobel Symposium 129.

\section{References}

[1] For a review see for example: S. F. King, Rept. Prog. Phys. 67 (2004) 107 arXiv:hep-ph/0310204, and references therein.

[2] P. Minkowski, Phys. Lett. B 67 (1977) 421; T. Yanagida in Proc. of the Workshop on Unified Theory and Baryon Number of the Universe, KEK, Japan, 1979; S.L.Glashow, Cargese Lectures (1979); M. Gell-Mann, P. Ramond and R. Slansky in Sanibel Talk, CALT-68-709, Feb 1979, and in Supergravity (North Holland, Amsterdam 1979); R. N. Mohapatra and G. Senjanovic, Phys. Rev. Lett. 44 (1980) 912; J. Schechter and J. W. F. Valle, Phys. Rev. D 22 (1980) 2227.

[3] S. F. King, Phys. Lett. B 439 (1998) 350 arXiv:hep-ph/9806440; S. F. King, Nucl. Phys. B 562 (1999) 57 arXiv:hep-ph/9904210; S. F. King, Nucl. Phys. B 576 (2000) 85 arXiv:hep-ph/9912492; S. F. King, JHEP 0209 (2002) 011 arXiv:hep-ph/0204360.

[4] S. Antusch and S. F. King, arXiv:hep-ph/0402121

[5] S. Antusch and S. F. King, Phys. Lett. B 591 (2004) 104 arXiv:hep-ph/0403053.

[6] S. Antusch and S. F. King, arXiv:hep-ph/0405272

[7] S. Antusch and S. F. King, Phys. Lett. B 597 (2004) 199 arXiv:hep-ph/0405093.

[8] G. Lazarides, Q. Shafi and C. Wetterich, Nucl. Phys. B 181 (1981) 287; R. N. Mohapatra and G. Senjanović, Phys. Rev. D23 (1981), 165; C. Wetterich, Nucl. Phys. B187 (1981), 343.

[9] T. Blazek and S. F. King, Phys. Lett. B 518 (2001) 109 arXiv:hep-ph/0105005; T. Blazek and S. F. King, Nucl. Phys. B 662 (2003) 359 arXiv:hep-ph/0211368.

[10] P. H. Frampton, S. L. Glashow and T. Yanagida, Phys. Lett. B 548 (2002) 119 arXiv:hep-ph/0208157; M. Raidal and A. Strumia, Phys. Lett. B 553 (2003) 72 arXiv:hep-ph/0210021; S. F. King, Phys. Rev. D 67 (2003) 113010 arXiv:hep-ph/0211228. A. Ibarra and G. G. Ross, arXiv:hep-ph/0312138.

[11] S. F. King and M. Oliveira, Phys. Rev. D 63 (2001) 095004 arXiv:hep-ph/0009287; T. Blazek, S. F. King and J. K. Parry, JHEP 0305 (2003) 016 arXiv:hep-ph/0303192.

[12] R. Barbieri, P. Creminelli and A. Romanino, Nucl. Phys. B 559 (1999) 17 arXiv:hep-ph/9903460; S. Raby, Phys. Lett. B 561 (2003) 119 arXiv:hep-ph/0302027.

[13] S. F. King and G. G. Ross, "Fermion masses and mixing angles from SU(3) family symmetry," Phys. Lett. B 520 (2001) 243 arXiv:hep-ph/0108112;

[14] S. F. King and N. N. Singh, Nucl. Phys. B 591 (2000) 3 arXiv:hep-ph/0006229; S. Antusch, these proceedings, arXiv:hep-ph/0409229 
[15] J. R. Ellis, M. Raidal and T. Yanagida, Phys. Lett. B 581 (2004) 9 arXiv:hep-ph/0303242; P. H. Chankowski, J. R. Ellis, S. Pokorski, M. Raidal and K. Turzynski, arXiv:hep-ph/0403180

[16] S. Antusch, M. Bastero-Gil, S. F. King and Q. Shafi, arXiv:hep-ph/0411298

[17] S. F. King and G. G. Ross, "Fermion Masses and Mixing Angles from SU(3) Family Symmetry and Unification," arXiv:hep-ph/0307190

[18] R. G. Roberts, A. Romanino, G. G. Ross and L. Velasco-Sevilla, Nucl. Phys. B 615 (2001) 358 arXiv:hep-ph/0104088.

[19] H. Georgi and C. Jarlskog, Phys. Lett. B 86 (1979) 297.

[20] M. Fukugita and T. Yanagida, Phys. Lett. B174 (1986) 45.

[21] M. Hirsch and S. F. King, Phys. Rev. D 64 (2001) 113005 arXiv:hep-ph/0107014.

[22] S. F. King, Phys. Rev. D 67 (2003) 113010 arXiv:hep-ph/0211228.

[23] T. Hambye and G. Senjanovic, Phys. Lett. B 582 (2004) 73 arXiv:hep-ph/0307237. 